\title{
PHYTOPLANCTON ET PRODUCTION PRIMAIRE DE LA RIVIERE LOT
}

\author{
par J. CAPBLANCQ ${ }^{1}$ et A. DAUTA ${ }^{1}$
}

1) Près de 190 espèces d'algues ont été identifiées dans le phytoplancton de la rivière Lot ; les Diatomées et les Chlorophycées dominent.

2) Les plus fortes biomasses ( 5 à $15 \mathrm{~g}$ de poids frais par $\mathrm{m}^{3}$ ) apparaissent dans les grands réservoirs de l'amont et dans la zone canalisée de la partie inférieure de la rivière lors de l'étiage estival. Aux très faibles débits sont associées des proliférations de Chlorophycées et une stratification verticale.

3) L'analyse des profils de photosynthèse, mesurée à l'aide de ${ }^{14} \mathrm{C}$, montre que la production photosynthétique par unité de surface $(\Sigma A)$ est fonction de la capacité photosynthétique du phytoplancton (Pmax), de l'extinction de la lumière $(\alpha)$ et du logarithme du quotient énergie lumineuse incidente/intensité du début de saturation de la photosynthèse par la lumière ( $\left.\mathrm{I}^{\prime} \mathrm{o} / \mathrm{Ik}\right)$. Pmax semble être déterminé par l'abondance des Chlorophycées nanoplanctoniques, elle-même liée à l'augmentation des températures et à la diminution du débit. Les variations de $\alpha$ suivent celles de l'abondance des matières en suspension où les algues n'interviennent que pour une faible proportion.

4) La production primaire du Lot moyen et inférieur a été estimée à $275 \mathrm{~g}$ $\mathrm{C} / \mathrm{m}^{2} /$ an dont près de $80 \%$ correspond aux trois mois d'été.

5) L'étude d'une masse d'eau au cours de son déplacement montre qu'une grande part $(80 \%)$ du carbone assimilé est perdue par respiration. Cette respiration représente moins de la moitié de l'oxygène consommé. La production extracellulaire est importante en été.

\section{Phytoplankton and Primary Production of the River Lot.}

1. Nearly 190 species of algae have been identified in the phytoplankton of the River Lot; Diatoms and Chlorophyceae dominate.

2. The highest biomasses $\left(5-15 \mathrm{~g} / \mathrm{m}^{3}\right.$ fresh weight) were found in the large upstream reservoirs and in the lower canalised part of the river under low flow conditions in summer. Chlorophyceae became abundant at very low flows and vertical stratification was evident.

3. Analysis of the photosynthetic profiles, measured with the ${ }^{14} \mathrm{C}$ method shows that integral photosynthesis $(\Sigma \mathrm{A})$ is a function of the photosynthetic capacity of the phytoplankton (Pmax), the extinction of light $(\alpha)$ and the log. of the quotient, surface-penetrating irradiance/irradiance at onset of light saturation of photosynthesis (I'o/Ik). Pmax is apparently determined by the abundance of nanoplanktonic Chlorophyceae, which are themselves influenced by temperature increases and decreases in flow. Variations in $\alpha$ follow variations in the quantity of suspended material in which algae play only a small role.

4. The primary production of the middle and lower Lot was estimated to be $275 \mathrm{~g} \mathrm{C} / \mathrm{m}^{2} /$ year, of which nearly $80 \%$ was produced in the three summer months.

5. The study of a moving mass of water shows that a large part $(80 \%)$ of the carbon assimilated is lost by respiration. This respiration represents less than half of the oxygen consumed. Extracellular production is important in summer.

1. Laboratoire d'Hydrobiologie (ERA 702), Université Paul-Sabatier, 118, route de Narbonne, 31077 Toulouse Cedex. 
Suite à divers aménagements, le cours de nombreuses rivières a été transformé en une succession de biefs à courant lent, fréquemment enrichis en substances minérales par les activités humaines. Ces conditions favorisent le développement de populations d'algues planctoniques. Longtemps moins étudié que celui des lacs, le plancton des rivières (ou potamoplancton) fait l'objet de recherches de plus en plus nombreuses. Certains résultats figurent dans les études hydrobiologiques de fleuves comme le Rhin (Czernin-Chudenitz 1958), le Danube (Szemes 1967), le Missouri (Berner 1951), le Nil (Prouse et Talling 1958), la Tamise (Mann et al. 1972) ou de retenues construites sur les rivières (Brook et Rzoska 1954, Wright 1954, Cushing 1964, Javornicky 1966, Cowel 1970, Young et al. 1972, Popova et al. 1972, Rai 1974). D'autres travaux portent plus spécialement sur le phytoplancton : études floristiques (Witford et Schumacker 1963, Egborge 1973), écologiques (Greenberg 1964, Czernin-Chudenitz 1966, Weber et Moore 1967, Venkateswarlu 1969, Lack 1971) ou mesures de production (Ertl et Juris 1967, Dvihally 1975, Sivko et al. 1972).

Les données publiées sur les rivières françaises (Des Cilleuls 1928, André 1926, Germain 1936, Lefèvre 1943, Manguin 1949, Tuffery 1971, Pierre 1972) sont, dans leur majorité, assez anciennes et essentiellement qualitatives. Le présent travail s'appuie sur les résultats de recherches quantitatives entreprises depuis 1972 dans la rivière Lot (Bordes et al., 1973, C.T.G.R.E.F. 1974, Dauta 1975, Denat 1977) et dont le but était de définir la structure du peuplement phytoplanctonique et les facteurs responsables du développement des algues.

\section{1. - MATERIEL ET METHODES}

Parmi les 23 stations étudiées par Dauta (1975) sur le cours du Lot, nous avons retenu ici 7 stations, les plus représentatives (fig. 1). Ce sont :

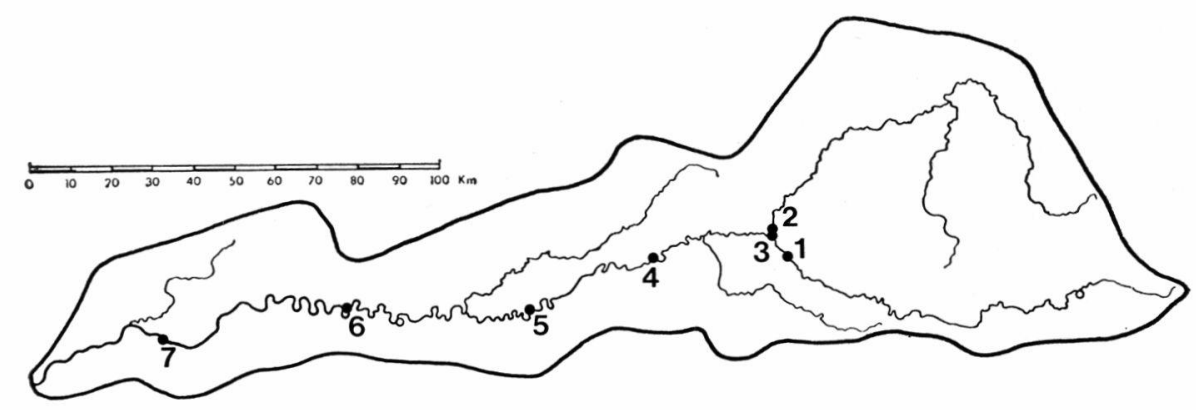

FIg. 1. - Carte du Lot et stations de mesure. 
station 1 : réservoir de Golinhac sur le Lot supérieur (km 162)

station 2: Lot à Entraygues en amont du confluent avec la Truyère (km 178)

station 3: Truyère à Entraygues

station 4 : Lot en amont du confluent avec le Riou-Mort (km 222)

station 5 : réservoir de Cajarc, au début de la traversée des Causses (km 271)

station 6: réservoir de Luzech sur le Lot inférieur ( $\mathrm{km} \mathrm{358)}$

station 7 : réservoir de Saint-Sylvestre, $60 \mathrm{~km}$ avant le confluent avec la Garonne ( $\mathrm{km} \mathrm{433).}$

Ces trois derniers réservoirs fonctionnent au fil de l'eau.

Des prélèvements mensuels ont été réalisés dans chaque station pour les comptages d'algues et les dosages de chlorophylle. Les cellules ont été dénombrées à l'aide d'un microscope inversé selon la technique décrite par Utermöhl (1958) et la biomasse évaluée par le calcul du biovolume. Les pigments chlorophylliens ont été dosés par spectrophotométrie sur des extraits acétoniques et leur concentration calculée à l'aide des équations de Lorenzen (1967).

L'activité photosynthétique du phytoplancton a été mesurée par la technique du ${ }^{1.4} \mathrm{C}$ dans le bief de Puy-l'Evêque, $34 \mathrm{~km}$ en aval de la station 6, de juin 1972 à août 1973 (C.T.G.R.E.F. 1974) et dans le réservoir de Cajarc (station 5) de février 1975 à février 1976 (Denat 1977). Des flacons clairs et opaques enrichis de ${ }^{14} \mathrm{CO}_{3} \mathrm{NaH}$ ont incubé à diverses profondeurs pendant 4 à 6 heures au milieu de la journée ; en fin d'expérience, la radioactivité des algues retenues sur une membrane filtrante de porosité $0,45 \mu \mathrm{m}$ et desséchées sur gel de silice a été mesurée à l'aide d'un compteur GM (FD1, Saphymo).

L'énergie lumineuse incidente pendant les jours d'expérience a été mesurée en 1975 par la méthode de l'anthracène (Marquis et Yelenosky 1962) étalonnée en référence aux valeurs fournies par un solarigraphe. La transmission dans l'eau des radiations actives pour la photosynthèse $\left(E_{400^{-700}}\right)$ a été calculée à partir de mesures photométriques réalisées à l'aide d'une cellule photoélectrique immergeable équipée de filtres RG2, VG9, BG12 (Schott, Mainz) et reliée à un milliampèremètre. Des mesures simultanées de transparence au disque de Secchi montrent qu'à la profondeur de limite de visibilité, l'intensité des radiations est égale en moyenne à $16 \pm 1,2 \%$ de l'intensité de surface $\left(\mathrm{I}^{\prime}{ }_{0}\right)$. Ce rapport, très voisin de celui que donne la relation de Jones et Wills (1956), permet d'estimer le coefficient d'extinction à partir des mesures de transparence.

Les caractères physico-chimiques de la rivière sont exposés par ailleurs (Tourenq et al. 1978, Capblancq et Tourenq 1978). 


\section{2. - PHYTOPLANCTON}

\section{1. - Composition du peuplement}

Près de 190 espèces et variétés ont été identifiées pour l'ensemble du Lot (tableau I). Les Diatomées (106 espèces) et les Euchlorophycées (49 espèces) sont les deux groupes les mieux représentés.

La diversité spécifique des Diatomées résulte de la présence d'un grand nombre d'espèces benthiques et épiphytiques en dérive. Les formes planctoniques comportent une quinzaine d'espèces seulement parmi lesquelles Fragillaria crotonensis, Melosira varians, Melosira granulata, Cyclotella sp., Stephanodiscus sp. dominent.

Theleau I. - Liste des espèces d'algues du phytoplancton du Lot. * : formes planctoniques.

EUCHLOROPHYCÉES

* Carteria simplex Pascher

* Chlamydomonas sp.

* Gonium sp.

* Lobomonas rostrata Hazen

* Pandorina morum (Muller) Bory

* Eudorina unicocca G.M. Smith

* Eudorina elegans Ehr.

* Pleodorina sp.

* Volvox globator (Linne) Ehr.

* Volvox aureus Ehr.

* Sphaerollepsis gloeocystiformis (Diel) Gerl.

* Torakomonas sp.

* Chlorococcum infusionum (Schrank) Meneghini

* Tetraedron minimum (Al. Braun) Hansgirg

* Chlorella sp.

* Chodatella quadriseta Lemm.

* Selenastrum bibraianum Reinsch

* Selenastrum minutum (Naeg.) Collins

* Ankistrodesmus falcatus (Corda) Ralfs

* Ankistrodesmus falcatus $v$. acicularis A. Braun

* Ankistrodesmus convolutus v. minutus (Näg) Rab.

* Ankistrodesmus sp.

Characium augustum A. Braun

* Micractinium pusillum Fres.

* Botryococcus braunii Kutz.

* Dictyosphearium sp.

* Coelastrum cambricum. Archer

* Coelastrum reticulatum (Dangeart) Senn.

* Coelastrum microporum Nag.

* Crucigenia tetrapedia Kirchner

* Scenedesmus quadricauda (Turpin) Breb.

* Scenedesmus quadricauda var. maximus W. et G. West.

* Scenedesmus nanus Chod.

* Scenedesmus bicaudatus Hansgirg

* Scenedesmus ecornis (Ralfs.) Chod.

* Scenedesmus acuminatus (Lagerh.) Chod.

* Scenedesmus spinosus Chod.

* Scenedesmus acutus (Meyen) Chod. 
* Scenedesmus falcatus Chod.

* Actinodesmus hantzschii Lagerh.

* Tetrallantos lagerheimii Teiling

* Golenkinia radiata Chod.

* Pediastrum tetras (Ehr.) Ralffs

* Pediastrum clathrarum Schroeter

* Pediastrum simplex Meyen

* Pediatrum duplex Meyen

* Pediastrum boryanum (Turpin) Meneghini

* Sphoerocystis sp.

* Westella botryoïdes (West) De Wyld

\section{ZYGOPHYCÉES}

Zygnema sp.

Mougeotia sp.

Closterium sp.

Euastrum sp.

Micrasterias sp.

Cosmarium sp.

Staurastrum sp.

Staurodesmus sp.

Spondylosium sp.

\section{ChrysophycéES}

* Chromulina sp.

* Chrysococcus sp.

* Kephyrion sp. Hydrurus foetidus (Vill.) Trev.

* Ochromonas sp.

* Uroglena sp.

* Uroglena nygardii Bourr.

\section{Diatomées}

* Melosira varians C. A. Agardh

* Melosira granulata (Ehr.) Ralfs

* Melosira granulata var. angustissima Mull.

* Cyclotella sp.

* Stephanodiscus sp.

* Rhizosolenia eriensis H.L. Smith

* Attheya zachariasi Brun

* Tabellaria fenestrata (Lyngb.) Kutz.

* Tabellaria flocculosa (Roth.) Kutz.

Diatoma vulgare Bory

Diatoma vulgare var. ovalis (Fricke) Hust.

Diatoma vulgare var. producta Brun.

Diatoma vulgare var. constricta Grun.

Diatoma vulgare var. capitulata Grun.

Meridion circulare Agardh.

Ceratoneis arcus Kutz.

Ceratoneis arcus var. amphyoxis (Rabh.)

Fragillaria intermedia Grun.

* Fragillaria crotonensis Kitton

Fragillaria construens (Ehr.) Grun.

Fragillaria construens var. binodis (Ehr.) Grun.

Fragillaria pinnata Ehr.

Fragillaria virescens Ralfs.

* Asterionella gracillima (Hantzsch) Heiberg

* Synedra ulna (Nitzsch) Ehr.

* Synedra ulna var. ramesi (Heribaud et Peragallo)

* Synedra ulna var. danica (Kutz.) Grun. 
Synedra rumpens Kutz.

Synedra rumpens var. scotica Grun.

Synedra affinis Kutz.

Synedra pulchella var. lanceolata O'Meara

Synedra vaucheriae Kutz.

Synedra parasitica var. subconstricta Grun.

Eunotia robusta Ralfs.

Eunotia praerupta Ehr.

Eunotia pectinalis var. ventralis (Ehr.) Hust.

Cocconeis placentula Ehr.

Cocconeis placentula var. euglyphta (Ehr.) Clève

Achnanthes minutissima var. cryptocephala Grun.

Achnanthes marginulata Grun.

Achnanthes lanceolata Breb.

Achnanthes lanceolata var. rostrata Hust.

Achnanthes clevei var. rostrata Hust.

Achnanthes lapponica Hust.

Rhoicosphenia curvata (Kutz.) Grun.

Frustulia rhomboïdes var. saxonica (Rabh.) De Toni

Gyrosigma spencerii var. nodifera Grun.

Gyrosigma acuminatum (Kutz.) Rabh.

Caloneis silicula (Ehr.) Clève

Neidium affine var. humerus (Ehr.) Clève

Diploneis ovalis (Hilse) Clève

Diploneis puella (Schumann) Clève

Stauroneis phoenicenteron Ehr.

Stauroneis legumen Ehr.

Navicula cuspidata Kutz.

Navicula minima Grun.

Navicula mutica Kutz.

Navicula pupula Kutz.

Navicula pupula var. capitata Hust.

Navicula pupula var. elliptica Hust.

Navicula cryptocephala Kutz.

Navicula cryptocephala var. intermedia Grun.

Navicula cryptocephala var. veneta (Kutz.) Grun.

Navicula viridula Kutz.

Navicula radiosa Kutz.

Navicula gracilis Ehr.

Navicula mutica var. nivalis (Ehr.) Hust.

Navicula exigua Gregory

Navicula hungarica var. capitata Grun.

Navicula dicephala var. elginensis (Grég.) Clève

Pinnularia interrupta W. Smith

Pinnularia mesolepta (Ehr.) W. Smith

Pinnularia braunii var. amphicephala (A. Mayer) Hust.

Pinnularia acrospheria Breb.

Amphiprora ornata Bayley

Amphora ovalis Kutz.

Cymbella amphicephala Nag.

Cymbella naviculiformis Auerswald

Cymbella cuspidata Kutz.

Cymbella prostrata (Berkeley) Clève

Cymbella ventricosa Kutz.

Cymbella affinis Kutz.

Cymbella cistula (Hemprich) Grun.

Cymbella helvetica Kutz.

Cymbella tumida (Breb.) Van Heurck.

Gomphonema acuminatum var. coronata (Ehr.) W. Smith

Gomphonema parvulum (Kutz.) Grun.

Gomphonema angustatum var. producta Grun.

Gomphonema constrictum Ehr. 
Gomphonema bohemicum Reichelt et Fricke

Gomphonema olivaceum (Lyngb.) Kutz.

Gomphonema olivaceum var. minutissima Hust.

Hantzschia amphyoxis (Ehr.) Grun.

Nitzschia hungarica Grun.

Nitzschia sinuata var. tabellaria Grun.

Nitzschia recta Hantzsch

* Nitzschia dissipata (Kutz.) Grun.

Nitzschia hantzschiana Rabh.

Nitzschia amphibia Grun.

Nitzschia fonticola Grun.

* Nitzschia palea (Kutz.) W. Smith

Nitzschia sigmoidae (Ehr.) W. Smith

Nitzschia sinuata (Wi Smith) Grun.

Cymatopleura solea (Breb.) W. Smith

Cymatopleura elliptica var. nobilis (Hantzsch) Hust.

Surirella linearis W. Smith

Surirella linearis var. helvetica (Brun) Meister

Surirella capronii Breb.

* Surirella ovata Kutz.

\section{CRYPTOPHYCÉES}

* Cryptomonas marssonii Skuja

* Cryptomonas erosa Ehr.

* Cryptomonas ovata Ehr.

* Chroomonas minuta Skuja

\section{DINOPHYCÉES}

* Peridinium sp,

* Ceratium sp.

\section{EUGLENOPHYCEES}

* Euglena sp.

* Trachelomonas sp.

\section{CYANOPHYCÉES}

* Chroococcus dispersus (Keissl.) Lemm.

* Microcystis aeruginosa $\mathrm{Kg}$.

* Oscillatoria limnetica Lemm.

* Anabaena sp.

* Pseudanabaena sp.

* Aphanizomenon sp. Phormidium muscicola Naumann et H. Pestalozzi.

Parmi les Chlorophycées, les Chlorococcales sont les formes les mieux représentées. Il s'agit, pour la plupart, d'algues de petite taille dont certaines sont difficilement identifiables. Les plus abondantes sont Scenedesmus sp., Ankistrodesmus falcatus, Micractinium pusillum, Dictyosphaerium sp., Selenastrum sp., Chodatella quadriseta, Coelastrum microporum, $C$. reticulatum. Les Volvocales unicellulaires (Chlamydomonas sp.) ou coloniales (Eudorina sp, Gonium sp., Pandorina morum), sont également toutes représentées dans les prélèvements d'été. 
Les Chrysophycées (Chromulina sp., Ochromonas sp.), les Cryptophycées (Cryptomonas, Chroomonas minuta) et les Cyanophycées (Anabaena sp., Pseudanabaena galeata, Aphanizomenon, Microcystis aeruginosa) sont présentes parfois en quantités non négligeables; des proliférations de Cyanophycées avec formation de fleurs d'eau peuvent apparaître sporadiquement dans certaines zones du Lot.

\section{2. - Evolution spatio-temporelle du peuplement}

L'évolution saisonnière du peuplement phytoplanctonique (fig. 2) se caractérise par la formation de fortes biomasses en été, correspondant aux faibles débits d'étiage et aux températures élevées.

Diatomées et Euchlorophycées représentent en moyenne plus de $90 \%$ de la biomasse. Les Diatomées, toujours présentes, constituent plus de $98 \%$ de la biomasse en hiver et au printemps, un peu moins de $50 \%$ en été. Leur cycle présente une première poussée printanière et une seconde automnale, plus faible, en plus de croissances estivales irrégulières de Melosira granulata et de Fragillaria crotonensis. Les Euchlorophycées abondent en août et septembre, époque durant laquelle on note des proliférations de Chlorococcales et de Volvocales nanoplanctoniques lors des plus faibles débits d'étiage. A la même époque peuvent apparaître des fleurs d'eau à base de Zygophycées (Mougeotia sp.), ou de Cyanophycées (Microcystis, Anabaena, Aphanizomenon), le plus souvent originaires des grands réservoirs du Lot supérieur; ces fleurs d'eau ne se développent qu'à des débits très faibles en présence de températures très élevées. Les Cryptophycées ne constituent en moyenne que $14 \%$ de la biomasse et sont présentes d'avril à octobre. Numériquement bien représentées, les Chrysophycées ne forment jamais de biomasses importantes compte tenu de leur petite taille.

Faible en hiver à toutes les stations, la biomasse de phytoplancton augmente au printemps, atteignant 2 à $9 \mathrm{~g}$ de poids frais par $\mathrm{m}^{3}$. Les plus fortes biomasses apparaissent en été dans les réservoirs de l'amont (station 1) et dans le Lot canalisé (stations 5 à 7) où elles atteignent $15 \mathrm{~g}$ de P.F. $/ \mathrm{m}^{3}$. Il existe cependant des variations importantes d'une année à l'autre : ainsi à la station 5, la biomasse moyenne d'été est de $2,5 \mathrm{~g}$ de P.F./m³ en 1972, 12,6 $\mathrm{g}$ en 1973 et $5 \mathrm{~g}$ en 1975 (fig. 2).

Il existe une corrélation hautement significative entre le biovolume d'algues du phytoplancton et la quantité de pigments chlorophylliens : cette relation indique que le quotient entre la concentration en chlorophylle $a$ et les M.O. algales, estimées à partir du biovolume d'après les valeurs données par Nalewajko (1966) et Strathmann (1967) varie entre 0,8 et $1,4 \%$.

La comparaison des stations permet de distinguer quatre zones successives, de l'amont vers l'aval de la rivière : 

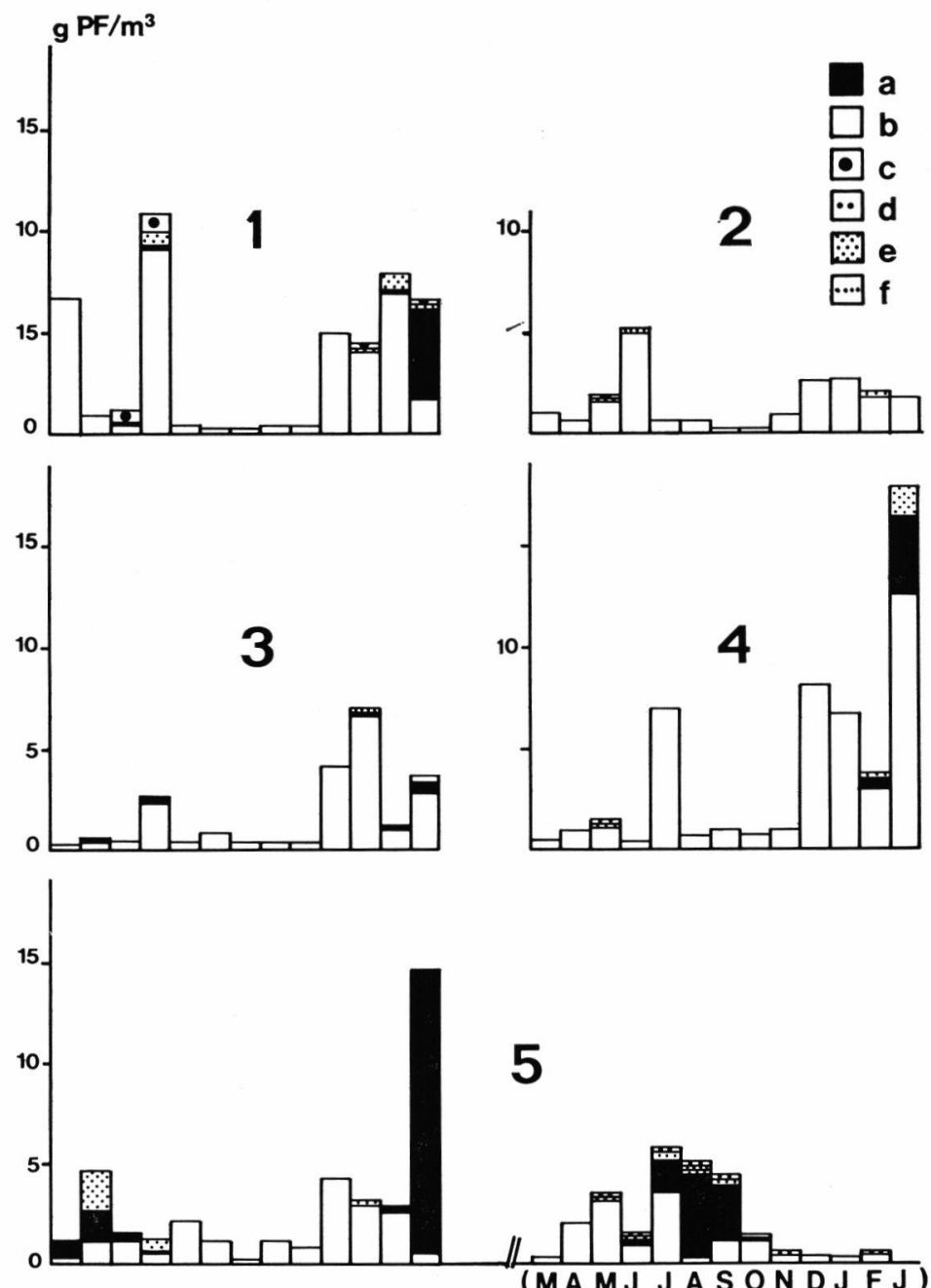

5
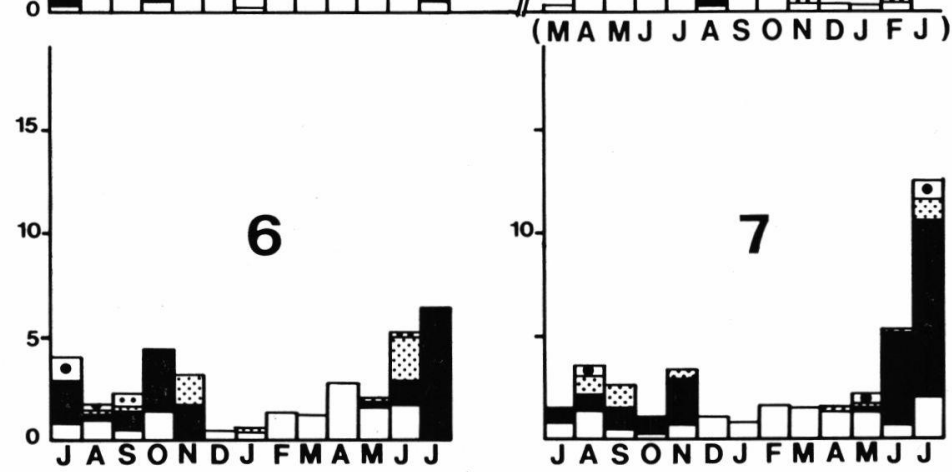

Fig. 2. - Variations saisonnières de la biomasse de phytoplancton aux différentes stations. a : Chlorophycées, b : Diatomées, c : Péridiniens, d: Chrysophycées, e : Cryptophycées, f: Cyanophycées. 
1. - Le cours supérieur à l'amont des réservoirs: la rivière se caractérise par un écoulement rapide et le peuplement microphytique est composé presque exclusivement de Diatomées (90 à $100 \%$ ) d'origine benthique ou périphytique. A l'entrée des premiers réservoirs (Castelnau), ce tychoplancton ne représente plus que $60 \%$ du nombre des cellules en suspension, le ralentissement du courant entraînant une sédimentation des formes benthiques et l'apparition de formes planctoniques.

2. - Les grands réservoirs de l'amont (Castelnau et Golinhac (station 1) sur le Lot, Couesque et Cambeyrac sur la Truyère) : le stockage de l'eau favorise le développement des communautés planctoniques. Dauta (1975) a montré que ces réservoirs constituaient des zones d'ensemencement en phytoplancton pour les zones d'aval, dans lesquelles les algues parviennent après un parcours relativement long $(43 \mathrm{~km})$ à courant assez rapide $\left(40 \mathrm{~cm} / \mathrm{s}\right.$ pour un débit de $\left.20 \mathrm{~m}^{3} / \mathrm{s}\right)$ entre les barrages et la zone canalisée. Si quelques espèces (Micractinium, Chodatella, Selenastrum, Ankistrodesmus) n'apparaissent que dans le cours inférieur, la majeure partie du plancton du Lot semble trouver son origine dans ces réservoirs de l'amont. Les populations des réservoirs du Lot et de la Truyère différant de manière sensible, le peuplement phytoplanctonique varie en aval selon l'origine des eaux lâchées. Dans ces réservoirs, la succession saisonnière du phytoplanc. ton diffère peu de celle observée pour l'ensemble du Lot et de celle décrite dans les lacs tempérés. Cependant, des fluctuations assez importantes de la biomasse d'algues sont liées aux caractéristiques hydrologiques (temps de rétention variable, turbulence).

3. - Le Lot moyen, entre Entraygues et Capdenac: sur une distance de $43 \mathrm{~km}$, l'écoulement ne rencontre aucun obstacle et le temps de séjour de la masse d'eau est relativement bref ( 28 heures pour un débit de $20 \mathrm{~m}^{3} / \mathrm{s}$ ). Cette zone (stations 2 à 4) est peu favorable à la croissance des algues dont la densité diminue sensiblement depuis la confluence du Lot et de la Truyère (station 3 ).

4. - Le Lot inférieur se présente comme une succession de bassins entrecoupés de nombreuses chaussées et barrages au fil l'eau (stations $5,6,7)$. La vitesse du courant y est très réduite en été $(0,07 \mathrm{~m} / \mathrm{s}$ pour $20 \mathrm{~m}^{3} / \mathrm{s}$ ) et les eaux stagnent fréquemment en amont des barrages; les temps de rétention de l'eau demeurent toutefois faibles. Dans cette zone, les algues benthiques en dérive ne contribuent en moyenne que pour 10 à $12 \%$ de la biomasse d'algues. Au contraire, les algues planctoniques issues des réservoirs de l'amont trouvent des conditions très favorables à leur croissance. En fait, la densité de phytoplancton est inversement proportionnelle au débit. Pour des débits inférieurs à $10 \mathrm{~m}^{3} / \mathrm{s}$ se développe une stratification du phytoplancton (fig. 3) et des propriétés chimiques liées au métabolisme des algues $\left(\mathrm{O}^{2}, \mathrm{NO}^{3}-\mathrm{N}\right.$, 
$\left.\mathrm{PO}_{4}-\mathrm{P}\right)$. C'est ainsi que les Diatomées sédimentent, les teneurs en phéopigments augmentent en profondeur et les formes mobiles telles que Volvocales, Chrysophycées et Cryptophycées se concentrent près de la surface.
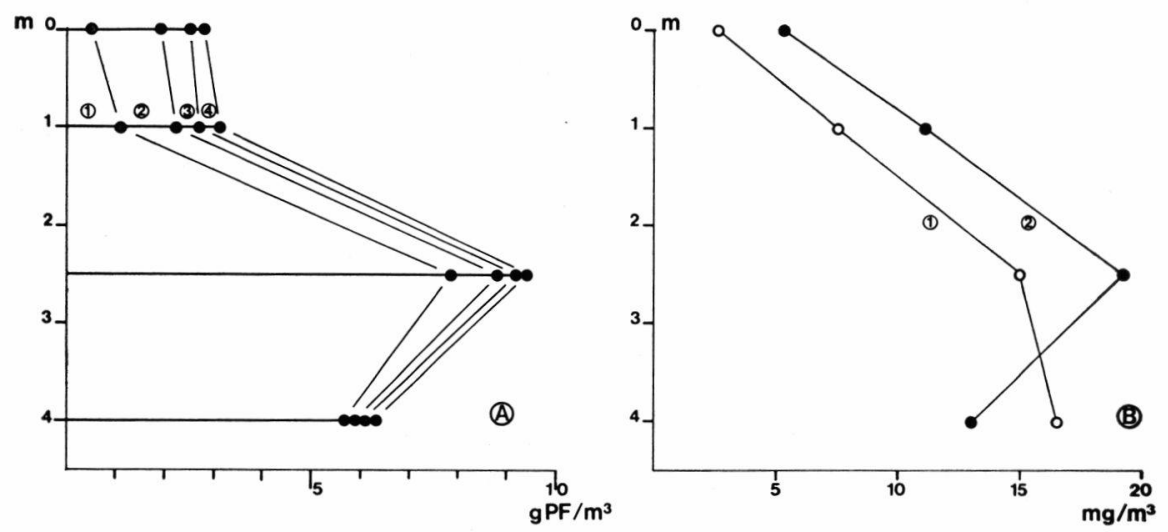

Fig. 3. - Distribution verticale de la biomasse de phytoplancton (A) et des pigments (B) dans le barrage de Cajarc le 24.07 .75 (débit $=7 \mathrm{~m}^{3} / \mathrm{s}$ ) ; fig. A: 1 : Diatomées, 2 : Chlorophycées, 3 : Cryptophycées, 4 : Chrysophycées ; fig. B : 1 : phéopigments, 2 : chlorophylle a.

\section{3. - PRODUCTION PRIMAIRE}

\section{1. - Activité photosynthétique dans les biefs de Cajarc et de Puy-l'Evêque}

Les eaux du Lot sont relativement turbides pendant toute l'année. Les radiations rouges et vertes pénètrent le plus profondément tandis que le bleu subit une extinction rapide. Ces propriétés optiques sont analogues à celles observées dans des eaux chargées en matières en suspension et en matières organiques dissoutes. La profondeur limite de la zone euphotique, c'est-à-dire celle où l'intensité des radiations actives pour la photosynthèse (PAR) est égale à $1 \%$ de celle de la surface, atteint 3,25 mètres en moyenne ; elle varie de 0,7 à $5,3 \mathrm{~m}$ pour l'ensemble des mesures (fig. 4). Ces variations sont essentiellement déterminées par la charge en matières en suspension (MES). Les matières organiques particulaires (MOP), mesurées par la perte au feu, peuvent constituer en été plus de $50 \%$ du poids des MES (fig. 4) ; la matière organique des algues planctoniques, estimée d'après le biovolume cellulaire et en admettant un rapport $\mathrm{MO} /$ poids frais de 0,3 pour les Chlorophycées et de 0,2 pour les Diatomées (Nalewajko 1966, Strathmann 1967), ne représente en moyenne que $30 \pm 15 \%\left(\mathrm{LC}_{0^{\prime}{ }_{05}}\right)$ des MOP pendant l'été. Ce résultat, analogue à celui que Mann et al. 

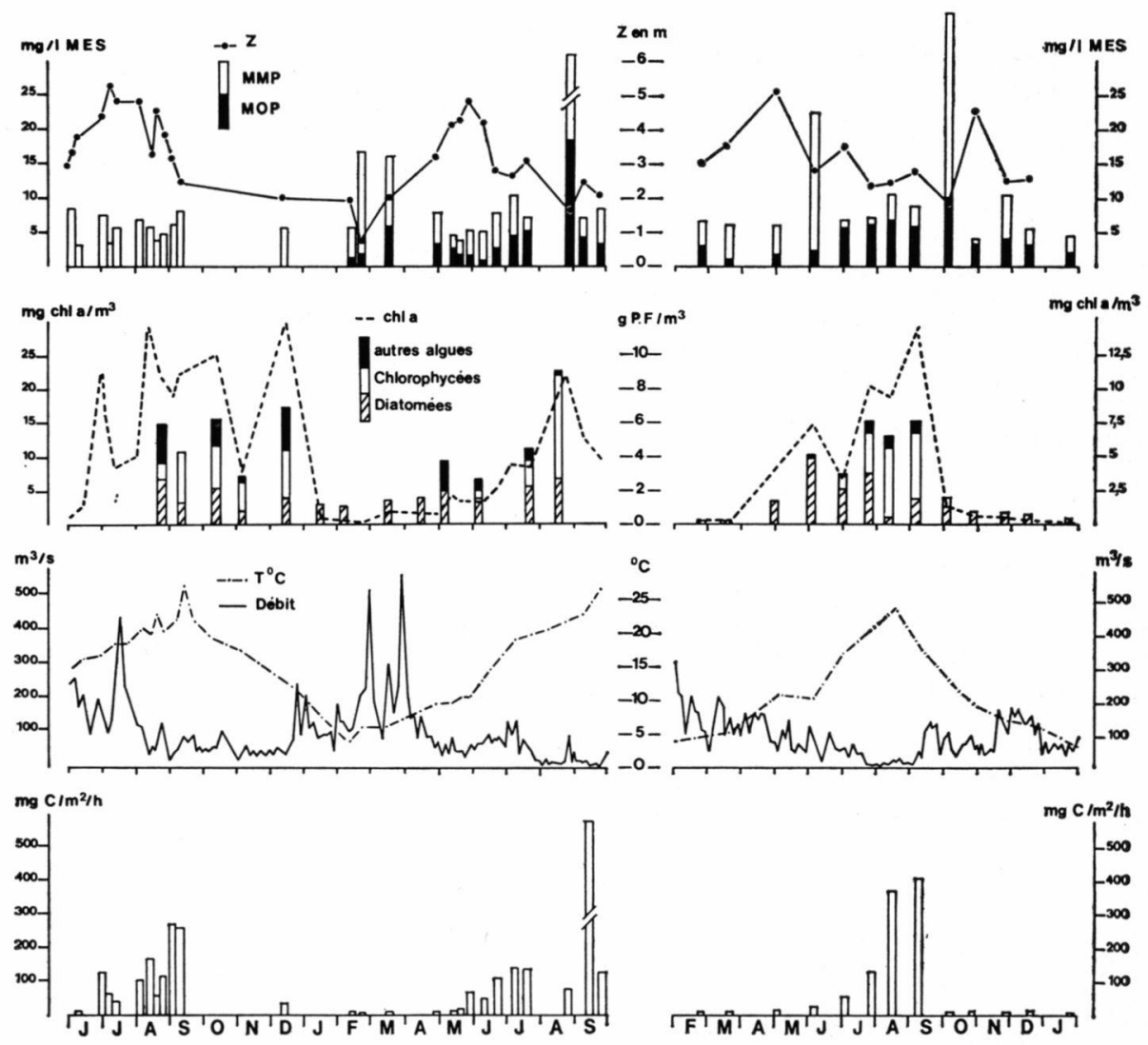

Fig. 4. - Variations de l'activité photosynthétique par unité de surface à midi $(\Sigma \mathrm{A})$ et de quelques facteurs du milieu dans les barrages de Puy-Lévêque (à gauche) et de Cajarc (à droite) : profondeur de la zone euphotique (Z), matières en suspension (MES) minérales (MMP) et organiques (MOP), biomasse de phytoplancton et concentration en chlorophylle a, température et débit.

(1972) rapportent pour la Tamise, indique que l'extinction de la lumière due aux algues planctoniques est sans doute relativement faible en regard de l'extinction due aux MES non vivantes. L'extinction variant selon la taille et la nature des particules, il n'existe pas de relation nette entre la charge en MES, la densité de phytoplancton et le coefficient d'extinction vertical.

Les profils verticaux de photosynthèse sont reproduits dans la figure 5. Ils sont généralement caractérisés par un optimum situé à une profondeur de 30 à $50 \mathrm{~cm}$ et indiquent une inhibition plus ou moins prononcée près de la surface, et, par suite, une saturation de la photosynthèse par la lumière. Puisque le phytoplancton se distribue de manière homogène avec la profondeur, les différences entre les divers profils résultent essentiellement des variations de pénétration 

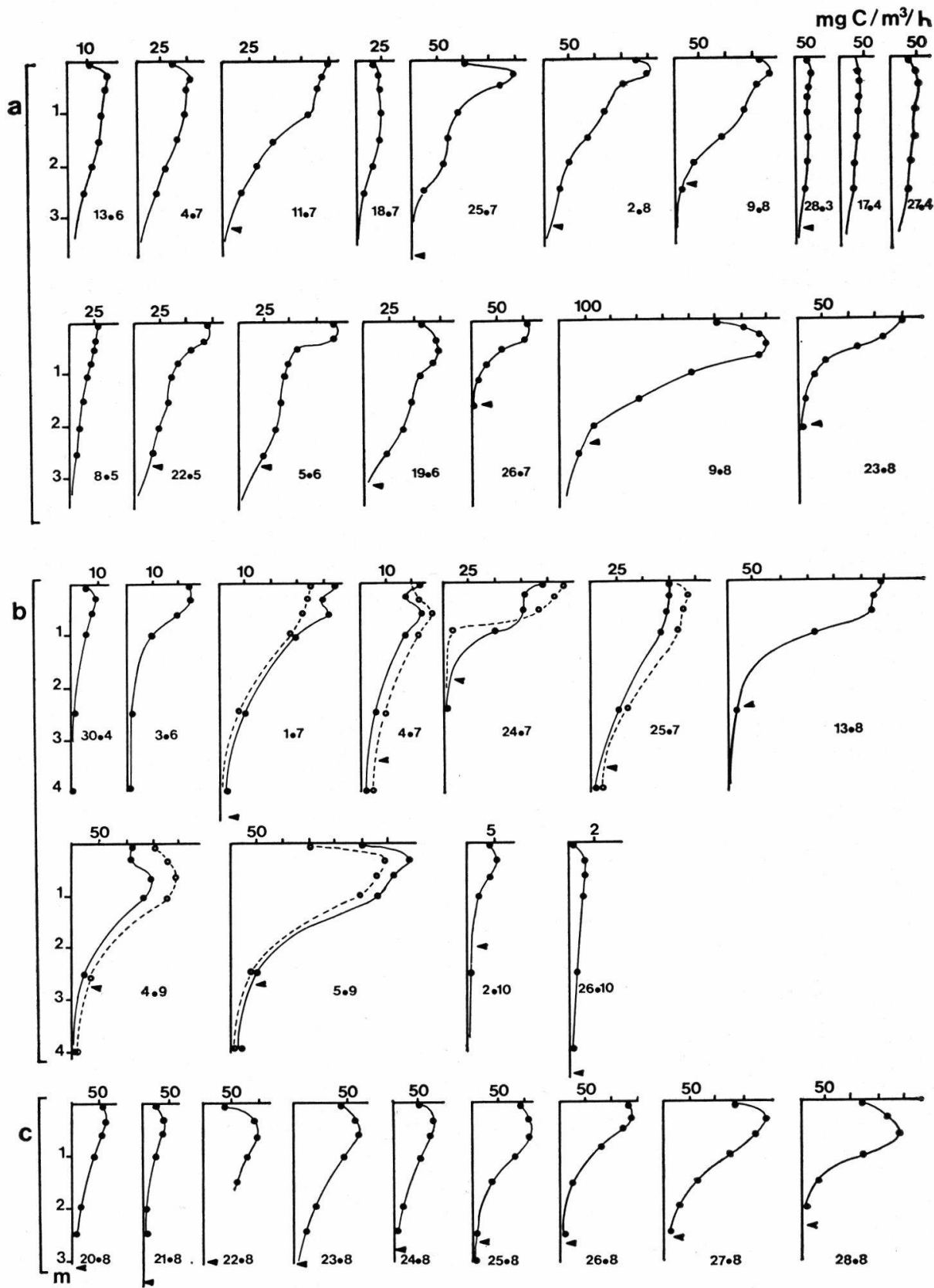

Fig. 5. - Profils verticaux de photosyntèse dans le bief de Puy-Lévêque (a) à deux stations du bief de Cajarc (b) et dans une masse d'eau durant son parcours vers l'aval (c). 
de la lumière. Un dénominateur commun à l'ensemble de ces courbes peut être obtenu en utilisant le concept de profondeur optique (Zod) défini par Talling (1957) et par Rodhe (1965). Les courbes d'assimilation relative (A/Amax) en fonction de Zod se ramènent alors à un modèle unique dans lequel les variations de la photosynthèse avec la profondeur sont une fonction de la lumière transmise. Une solution mathématique de ce modèle a été proposée par Talling (1957), et reprise par plusieurs auteurs (Vollenweider 1965, Rodhe 1965, Patten 1968, Fee 1973). Dans ce modèle basé sur l'équation semi-empirique de Smith (1936), la forme des courbes et ses variations sont déterminées essentiellement par deux paramètres :

- la capacité photosynthétique optimale (Pmax)

- l'intensité de début de saturation de la photosynthèse par la lumière, que Talling (1957) désigne par le symbole Ik.

- La capacité photosynthétique optimale (Pmax) varie de 0,22 à $35 \mathrm{mg} \mathrm{C} / \mathrm{mg} \mathrm{Chl} . \mathrm{a} /$ heure dans l'ensemble des mesures réalisées sur le Lot (fig. 6). Les valeurs les plus faibles correspondent à la saison hivernale et au début du printemps, lorsque les débits sont élevés et les températures basses. La biomasse de phytoplancton est alors généralement faible et composée uniquement de Diatomées parmi lesquelles les formes benthiques en dérive peuvent constituer $1 / 3$ du biovolume total (Dauta 1975, Denat 1977). Inversement, les fortes valeurs correspondent aux débits d'étiage et aux températures élevées de l'été, le peuplement étant alors composé en majorité de Chlorophycées et de Cryptophycées de petites tailles. Entre juin et octobre, la valeur moyenne de Pmax est de 8,4 $\pm 2,1 \mathrm{mg} \mathrm{C} / \mathrm{mg} \mathrm{Ch.a/h}\left(\mathrm{LC}_{0}{ }^{\circ}{ }^{2}\right)$, ce qui témoigne d'une activité photosynthétique assez élevée : à titre de comparaison, les valeurs de Pmax.max relevées pour les lacs mésotrophes à eutrophes des régions tempérées varient entre 3 et $12 \mathrm{mg} \mathrm{C} / \mathrm{mg}$ Ch.a/h. Il n'existe, par ailleurs, aucune relation inverse entre la capacité photosynthétique des algues et la biomasse de phytoplancton comme on en rencontre dans certains milieux (par exemple, Elster 1965, Findenegg 1965, Capblancq 1972). Par contre (fig. 6), les variations de Pmax dépendent étroitement de la nature du phytoplancton, elle-même liée au débit et à la température. En effet, aux faibles débits d'étiage, la température augmente et un nanoplancton à base de Chlorophycées (Scenedesmus, Ankistrodesmus, Coelastrum) domine le peuplement. Ce nanoplancton est beaucoup plus actif que le plancton de printemps composé de Diatomées originaires des grands barrages de l'amont.

- Ik peut être déterminé sur un graphique semi-logarithmique comme la profondeur optique correspondant à l'intersection du prolongement de la portion linéaire de la courbe de photosynthèse avec l'abscisse de Amax (Vollenweider 1965, Rodhe 1965). Selon l'équation 
de Smith (1936), cette intensité correspond à un taux de photosynthèse égal à 0,71 Amax.

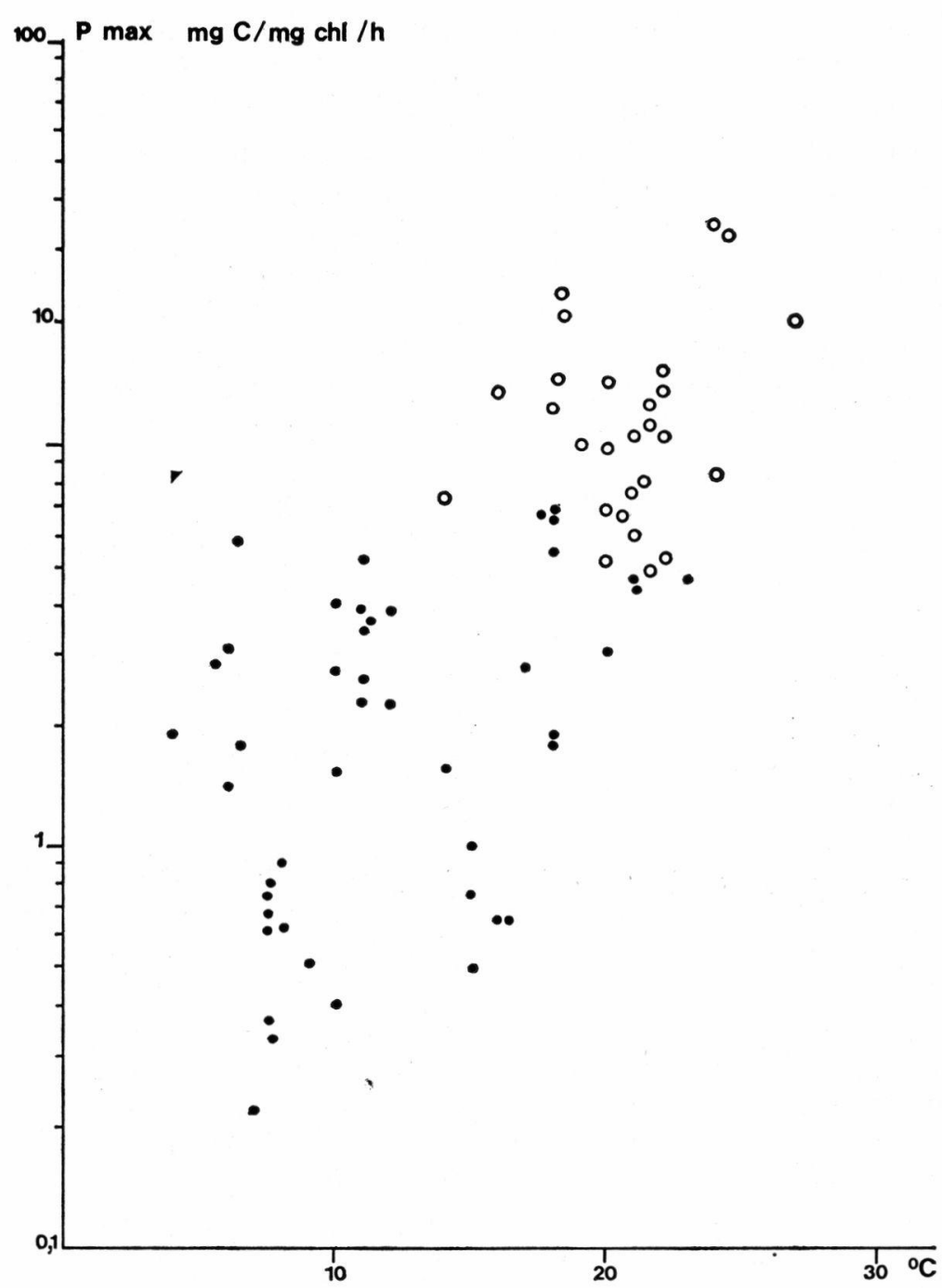

FIG. 6. - Relation entre la capacité photosynthétique optimale ( $\mathrm{P} \max$ ) et la température : les points noirs correspondant à un phytoplancton à base de Diatomées, les points blancs à une dominance de Chlorophycées $(>50 \%$ du biovolume total).

La détermination graphique de Ik sur nos profils de photosynthèse montre qu'à cette intensité le rapport $\mathrm{A} / \mathrm{Amax}$ est égal en moyenne à $0,77 \pm 0,04\left(\mathrm{LC}_{0^{\prime}{ }^{05}}\right)$, valeur très voisine de celle indiquée par Tal- 
ling (1965). En admettant avec cet auteur que Ik est l'intensité pour laquelle $\mathrm{A}=0,75$ Amax, les courbes de photosynthèse du Lot indiquent que :

1) Ik varie de 0,14 à $0,45 \mathrm{I}^{\prime}{ }_{0}$, avec une moyenne de $0,26 \pm 0,055 \mathrm{I}^{\prime}{ }_{0}$ $\left(\mathrm{LC}_{0^{\prime} 05}\right)$.

2) pour $\mathrm{I}=0,5 \mathrm{Ik}, \mathrm{A} / \mathrm{Amax}=0,47 \pm 0,035$.

Les expériences réalisées au cours de l'été 1975 indiquent, en outre, qu'en valeur absolue, Ik est de l'ordre de $3,7 \pm 1,1 \mathrm{cal} / \mathrm{cm}^{2} / \mathrm{h}$ en moyenne, avec des valeurs extrêmes de 1,1 et $6,7 \mathrm{cal} / \mathrm{cm}^{2} / \mathrm{h}$.

La figure 7, construite à partir des profils de photosynthèse obtenus entre mai et novembre à Cajarc, montre que la pente initiale $(\mathrm{K})$ des courbes photosynthèse-intensité lumineuse, qui est égale au quotient Pmax/Ik, est extrêmement variable : ainsi, $\mathrm{K}$ passe de $0,6 \mathrm{mg} \mathrm{C} / \mathrm{mg}$
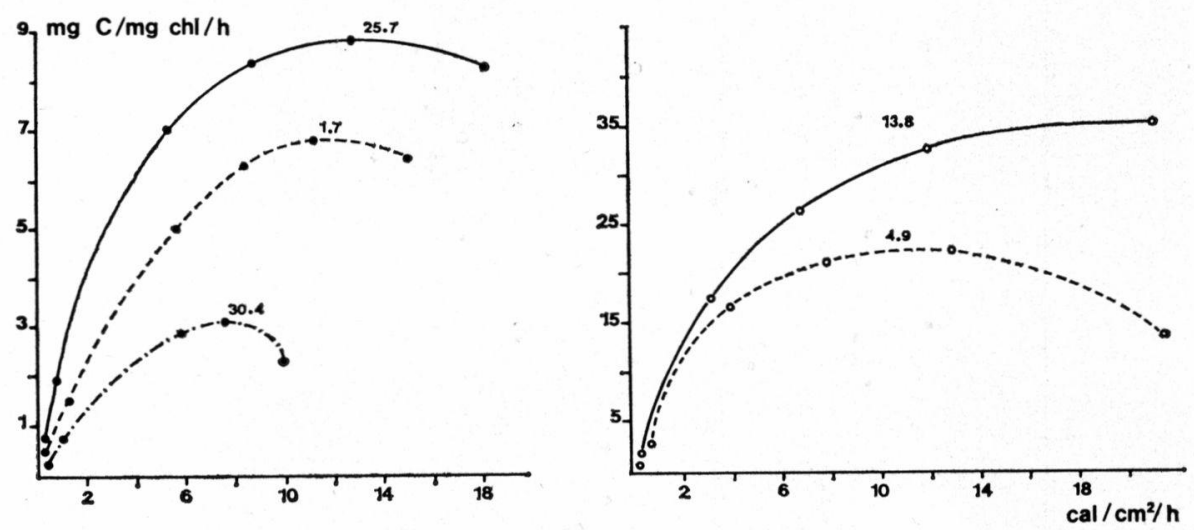

FIG. 7. - Courbes de photosynthèse en fonction de l'intensité lumineuse.

Chl.a/cal.cm ${ }^{-2} \mathrm{~h}^{-1}$ le 30 avril à 7,7 le 13 août ; les variations de $\mathrm{K}$ suivent celles de Pmax tandis que Ik reste relativement constant. L'efficacité de conversion de l'énergie lumineuse augmente donc comme Pmax, en même temps que la température et la proportion de Chlorophycées croissent.

Il existe une relation étroite entre l'activité photosynthétique de la zone euphotique ( $\Sigma \mathrm{A}$ ) et le quotient Amax/ $\alpha$ (fig. 8 a) ; l'intégration des profils de photosynthèse peut s'écrire, au seuil de probabilité $5 \%$ :

$$
\Sigma \mathrm{A}=\frac{\operatorname{Amax}}{\alpha} \times(2,21 \pm 0,15)
$$

$\alpha$ étant le coefficient d'extinction vertical de la lumière.

Le coefficient d'intégration des profils de photosynthèse, généralement voisin de 2,6 (Vollenweider 1965, Jewson 1976), est donc légère- 

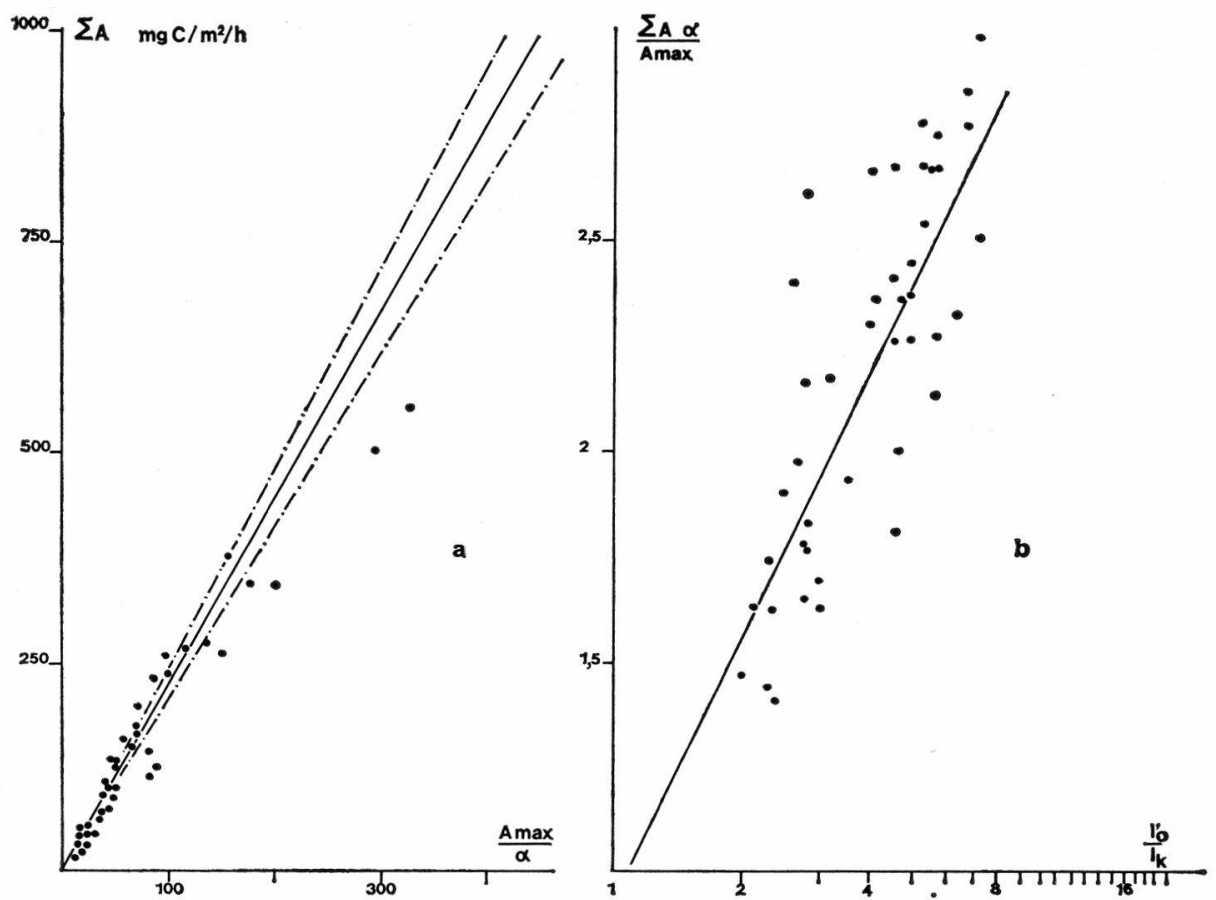

Fig. 8. - Variations de l'activité photosynthétique par unité de surface de plan d'eau $(\Sigma \mathrm{A})$ en fonction du rapport A max $/ \alpha$ (fig. 8 a) et relation avec le rapport $\mathrm{I}^{\prime} / \mathrm{I}_{\mathrm{k}}$ (fig. $8 \mathrm{~b}$ ).

ment plus faible dans le cas du Lot; la différence tient aux valeurs relativement élevées du paramètre Ik.

Dans la figure $8 \mathrm{~b}$ sont représentées les valeurs de $\frac{\Sigma A . \alpha}{A m a x}$ en fonction du logarithme du quotient $\mathrm{I}_{0}{ }_{0} / \mathrm{Ik}$; la liaison existant entre ces deux paramètres $(r=0,705$ pour 42 DL) est conforme à la solution de Talling (1957) qui décrit les variations de $\Sigma$ A comme une fonction de $\mathrm{I}^{\prime}{ }_{0} / \mathrm{Ik}$. Dans cette équation, $\mathrm{I}_{0}{ }_{0}$ et $\mathrm{Ik}$ intervenant par leur logarithme, leur variation influe moins sur la valeur de $\Sigma A$ que les variations de Amax; si Ik, Amax et $\alpha$ restent constants pendant toute la journée, les variations journalières de $\Sigma$ A suivront celles du logarithme de $I^{\prime}{ }_{0}$ (Talling 1971). Ce dernier point permet de calculer le taux journalier de photosynthèse à partir d'expériences de courte durée, en utilisant un facteur de conversion journalier (F) (Talling 1957) tel que :

$$
\mathrm{F}=\frac{\operatorname{Ln} \overline{\mathrm{I}}_{0}-\operatorname{Ln} 0,5 \mathrm{Ik}}{\operatorname{Ln} \mathrm{I}^{\prime}{ }_{0}-\operatorname{Ln} 0,5 \mathrm{Ik}} \times 0,9 \times \Delta \mathrm{t}
$$


où : $\overline{\mathrm{I}_{0}}=$ intensité lumineuse pendant le jour

$\mathrm{I}_{0}^{\prime}=$ intensité lumineuse pendant les heures d'exposition

$\Delta \mathrm{t}=$ durée du jour en heures.

Des mesures faites à Cajarc montrent que cette méthode de calcul fournit des valeurs proches de celles obtenues par sommation d'expériences successives réalisées entre le lever et le coucher du soleil. Les expériences de l'année 1975 montrent, en outre, que, exception faite de quelques journées très nuageuses d'hiver, $F$ varie entre 0,65 et $0,73 \Delta \mathrm{t}$.

Les valeurs de $\Sigma A$ ne diffèrent pas significativement entre les biefs de Cajarc et de Puy-l'Evêque. Le cycle saisonnier, étroitement lié au régime des débits, se caractérise par une faible production hivernale et un maximum estival (fig. 4). Ainsi, entre le début du mois d'octobre et le mois de mai, la production journalière moyenne est de $68 \mathrm{mg}$ $\mathrm{C} / \mathrm{m}^{2} / \mathrm{j}$ (valeurs extrêmes : 2 et 245 ) ; au printemps (mai, juin) elle passe à $745 \mathrm{mg} \mathrm{C} / \mathrm{m}^{2} / \mathrm{j}$ en moyenne (valeurs extrêmes : 235 et 1377 ). En été (juillet, août, septembre), la production moyenne atteint $2320 \mathrm{mg} \mathrm{C} / \mathrm{m}^{2} / \mathrm{j}$ (valeurs extrêmes : 504 et 10650 ). Pour l'ensemble de l'année, on peut donc estimer à $275 \mathrm{~g} \mathrm{C} / \mathrm{m}^{2} /$ an la production des biefs du Lot canalisé ; $78 \%$ de cette production correspond aux trois mois d'été. Une telle production s'apparente à celle des eaux à degré de trophie élevé (Vollenweider 1968).

\section{2. - Production extracellulaire}

Quelques travaux (Schindler 1970, Schindler et al. 1972, Lean 1972 et 1973) montrent que l'augmentation de la radio-activité par unité de volume d'eau lorsqu'on réduit le volume filtré d'échantillons d'eau

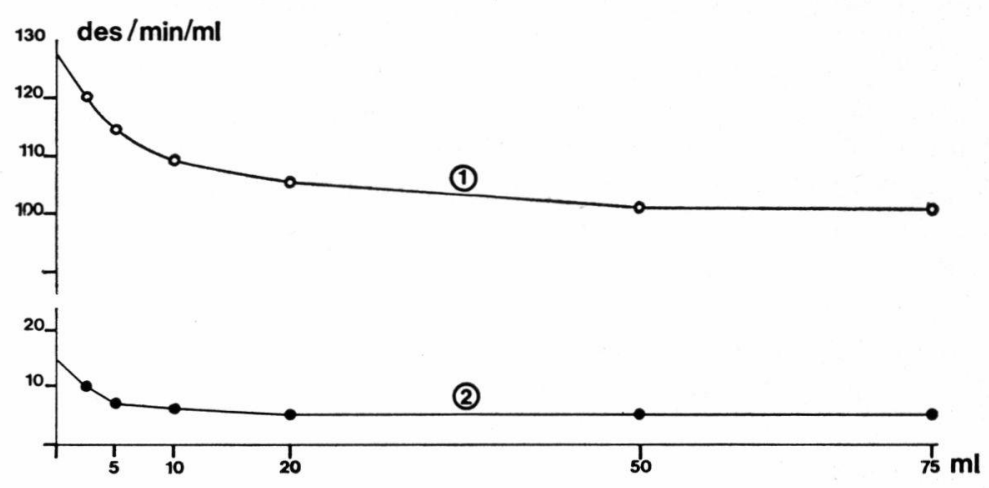

Fig. 9. - Relation entre l'activité retenue par le filtre et le volume d'échantillon filtré : 1 : flacon transparent après incubation; 2 : flacon témoin. 
incubée avec du ${ }^{14} \mathrm{C}$ (effet de Arthur et Rigler 1967) résulte de l'adsorption sur la membrane filtrante de matières organiques marquées. Les expériences réalisées par Lean (op. cit.) et Nalewajko et Schindler (1976) indiquent que ces matières organiques proviennent du métabo. lisme des algues (production extracellulaire) et sont constituées de grosses molécules de poids moléculaire $\geqslant 1500$. Ainsi, l'extrapolation des courbes de radio-activité en fonction du volume filtré (fig. 9) doivent permettre d'évaluer l'importance de cette production extracellulaire (Schindler 1970). De telles courbes ont été systématiquement établies pour les échantillons exposés à $-60 \mathrm{~cm}$ lors des expériences réalisées à Cajarc. Les résultats montrent une évolution saisonnière caractérisée par :

- une production extracellulaire pratiquement indécelable entre octobre et avril,

- des valeurs élevées entre juillet et septembre ; la radio-activité des produits extracellulaires adsorbés sur le filtre correspond en moyenne à $16,6 \%$ de l'assimilation photosynthétique totale, et varie de 4 à $40 \%$.

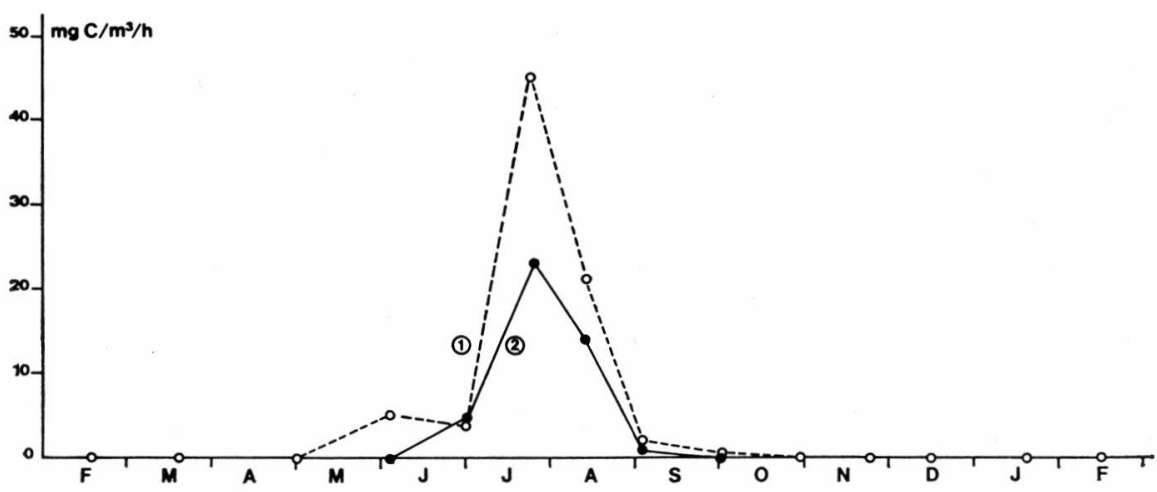

Fig. 10. - Evolution du taux d'assimilation de carbone à l'obscurité (1) et de la production extracellulaire (2) dans le réservoir de Cajarc.

On connaît assez mal les facteurs responsables de cette excrétion qui paraît relativement plus importante dans les couches superficielles des eaux oligotrophes. Il apparaît toutefois que les molécules organiques provenant de cette excrétion sont, sous l'action des bactéries, à l'origine d'une partie des MOP des eaux du Lot. En effet, les mesures effectuées à Cajarc montrent qu'il existe une correspondance entre les taux élevés de production extracellulaire et les teneurs élevées en MOP. De même, aux valeurs élevées de production extracellulaire correspondent des taux élevés d'assimilation hétérotrophe de ${ }^{14} \mathrm{C}$ (fig. 10) qui témoignent essentiellement de l'activité bactérienne. 


\section{4. - DYNAMIQUE}

\section{DU PHYTOPLANCTON DANS UNE MASSE D'EAU MARQUEE}

Cette étude réalisée entre le 20 et le 29 août 1975 avait pour but de suivre, en période d'étiage et de débit régularisé à $16-19 \mathrm{~m}^{3} / \mathrm{s}$, l'évolution du phytoplancton dans une masse d'eau durant son parcours vers l'aval. L'expérience a été réalisée entre Cajarc et Cahors sur une distance de $52 \mathrm{~km}$. L'eau a été marquée au départ (Cajarc) par de la Rhodamine, et les prélèvements réalisés matin et soir, dans des stations choisies en fonction de la progression de la masse d'eau marquée. Sur ces prélèvements ont été réalisés des comptages d'algues, des dosages de chlorophylle et des analyses chimiques. Le taux d'assimilation photosynthétique a été mesuré chaque jour entre 11 et $15 \mathrm{~h}$ sur des échantillons immergés à diverses profondeurs (fig. $5 \mathrm{c}$ ); parallèlement, la consommation d'oxygène a été déterminée à l'aide de flacons opaques immergés. Les résultats de ces mesures, analysées par Capblancq et Décamps (1977) sont résumés dans le tableau II et la figure 11.

En 10 jours, l'eau a parcouru $52 \mathrm{~km}$ à la vitesse moyenne de $6,5 \mathrm{~cm} / \mathrm{s}$. L'évolution du peuplement phytoplanctonique révèle :

- l'abondance des Chlorophycées (Scenedesmus, Ankistrodesmus, Coelastrum, Kirchneriella) qui composent 70 à $90 \%$ de la biomasse. Les Diatomées (Melosira, Fragillaria) et les Cyanophycées (Pseudanabaena, Coelosphaerium) ne constituent respectivement que 5 à $20 \%$, et 3 à $10 \%$ de la biomasse d'algues.

- Une croissance régulière du phytoplancton, traduite par les courbes d'évolution de la chlorophylle $a$, de la biomasse, et du nombre de cellules.

- La croissance du phytoplancton est essentiellement due à une espèce nanoplanctonique (Coelastrum reticulatum) dont la densité passe de $500 \varnothing / \mathrm{ml}\left(0,15 \mathrm{~g} \mathrm{PF} / \mathrm{m}^{3}\right)$ à $50000 \varnothing\left(5,2 \mathrm{~g} \mathrm{PF} / \mathrm{m}^{3}\right)$ en $52 \mathrm{~km}$, tandis que la densité des autres espèces reste pratiquement stable (fig. 11).

La relation établie entre biovolume d'algues et quantité de chlorophylle $a$ d'une part et les équations de Strathmann (1967) qui donnent le quota cellulaire des algues en carbone en fonction du volume des cellules d'autre part, a été utilisée pour estimer l'équivalent en carbone de la biomasse de phytoplancton. Les résultats (fig. 11) montrent que, pendant les 10 jours d'expérience, l'accroissement de la biomasse peut se décrire par une équation exponentielle :

$\mathrm{C}=\mathrm{C}_{0} \cdot \mathrm{e}^{0,0055 \mathrm{t}}$ où $\mathrm{C}_{0}$ et $\mathrm{C}$ sont l'équivalent carbone du phytoplancton au début de l'expérience $\left(t_{0}\right)$ et au temps $t$. La dérivée de cette équation exprime l'accroissement net de la biomasse, c'est-à-dire la production primaire nette (tableau II). 







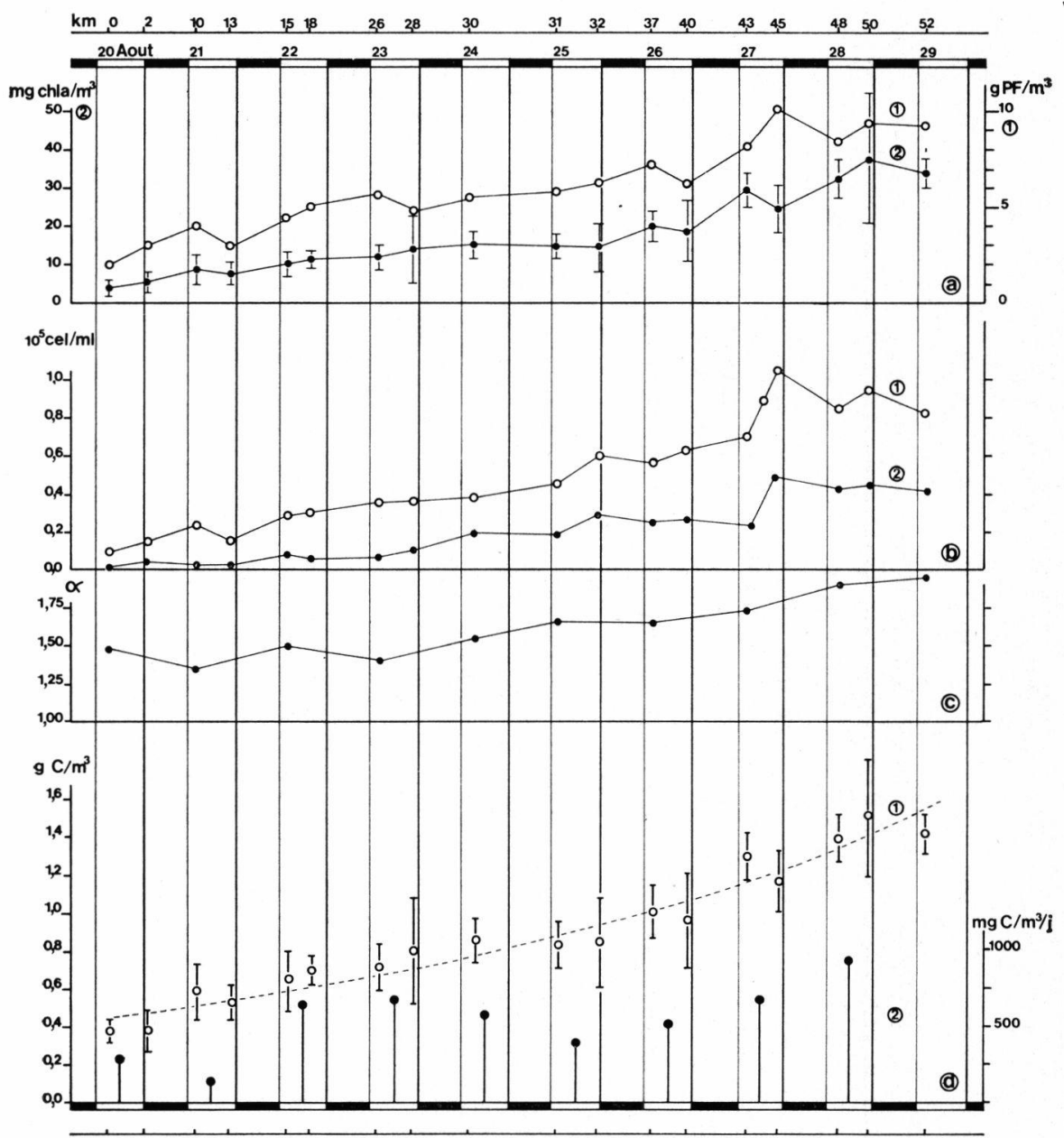

Fig. 11. - Evolution de la biomasse de phytoplancton (a, 1), de la quantité de chlorophylle a $(a, 2)$, du nombre de cellules total $(b, 1)$ et de Coelastrum reticulatum (b, 2), du coefficient d'extinction vertical (c), du carbone du phytoplancton $(\mathrm{d}, 1)$ et du taux journalier de photosynthèse $(\mathrm{d}, 2)$ dans une masse d'eau durant son parcours vers l'aval. La courbe en pointillés $(d, 1)$ représente l'équation $\mathrm{C}=\mathrm{C}_{0} \cdot \mathrm{e}^{0,0055 \mathrm{t}}$.

Comparées aux taux journaliers d'assimilation photosynthétique (A), les valeurs de $\mathrm{P}$ sont relativement faibles : ceci indique qu'une proportion élevée (environ $80 \%$ ) du carbone incorporé par photosynthèse est perdue par respiration et biodégradation (tableau II). On admet généralement que la méthode du ${ }^{14} \mathrm{C}$ mesure une valeur comprise entre l'assimilation photosynthétique nette (An) et l'assimilation brute $(\mathrm{Ab})$ pendant les heures d'éclairement; aux limites, la différence 
[A - P] représente donc soit les pertes nocturnes, soit les pertes en 24 heures.

Ces pertes par respiration et biodégradation ne représentent qu'une faible proportion du taux de consommation d'oxygène. Aux limites, le carbone assimilé par les algues et dégradé équivaut à $53 \%$ $(42-72 \%)$ et $24 \%(20-33 \%)$ de l'oxygène consommé. Ainsi, plus de $50 \%$ de l'oxygène consommé dans les flacons opaques résulte de la dégradation bactérienne des MOD et des MOP dont la teneur moyenne est respectivement de $9 \mathrm{mg} / \mathrm{l}$ et de $5,4 \mathrm{mg} / 1$ pendant la durée de l'expérience. Ce résultat est en accord avec ceux que Golterman (1971) rapporte pour les eaux d'un lac eutrophe.

\section{5. - DISCUSSION}

La comparaison du peuplement phytoplanctonique du Lot avec celui de rivières nord-américaines (Whitford et Schumacher 1963, Weber et Moore 1967, Soltero et al. 1975) ou européennes (Lack 1971) révèle une assez grande similitude sur le plan qualitatif et quantitatif. Les espèces dominant le phytoplancton du Lot se retrouvent également abondantes dans le phytoplancton des lacs mésotrophes à eutrophes des régions tempérées. Si l'on se réfère aux listes d'espèces indicatrices données par Liebmann $(1960,1962)$ et Turoboyski (1966) ou aux caractéristiques écologiques des Diatomées établies par Cholnoky (1968), le peuplement du Lot correspondrait à celui d'un milieu $\beta$ mésosaprobe. Cependant, Villegas et De Giner (1973) et Golowin (1971) ont souligné les difficultés liées à l'utilisation du phytoplancton comme indicateur biologique.

Il est à noter qu'en dépit du caractère relativement instable du milieu (variations brutales de débit et des niveaux, lâchers intermittents d'eau en provenance du Haut Lot et de la Truyère, rejets industriels et domestiques entraînant des variations des caractères chimiques) on retrouve sur l'ensemble du Lot la même succession saisonnière que dans de nombreux lacs. Les biomasses maximales apparaissent cependant l'été et correspondent aux plus faibles débits d'étiage.

Plusieurs auteurs (Wright 1954, Rodhe 1964, Popova et al. 1972, Egborge 1974) ont noté que le stockage de l'eau ou la réduction de la vitesse du courant favorisent la croissance et la persistance de formes planctoniques. Dans le Lot, les grandes retenues de la zone amont sont propices au développement du phytoplancton; elles constituent, par ailleurs, une source d'ensemencement pour la zone moyenne et inférieure où le ralentissement du courant par les chaussées et les barrages au fil de l'eau permet la reprise de la croissance des espèces planctoniques. Ce rôle des barrages est également démontré par 
Cushing (1954) qui note qu'entre l'amont et l'aval d'une retenue on passe d'un plancton composé d'algues en dérive, à un plancton vrai.

Une stratification du phytoplancton et des composantes chimiques liées à son métabolisme $\left(\mathrm{O}_{2}, \mathrm{NO}_{3}-\mathrm{N}, \mathrm{NH}_{4}-\mathrm{N}, \mathrm{PO}_{4}-\mathrm{P}\right)$ apparaît dans le Lot moyen et inférieur pour des débits de l'ordre de $10 \mathrm{~m}^{3} / \mathrm{s}$ (vitesse moyenne de 4 à $5 \mathrm{~cm} / \mathrm{s}$ ), en l'absence de gradient thermique perceptible. Ce phénomène, vraisemblablement lié à la faiblesse du courant, résulte d'une part de la sédimentation des Diatomées et d'autre part d'une migration des algues vertes vers les zones d'éclairement optimal dans lesquelles le taux de division cellulaire est accru. Le cas le plus fréquent est cependant une distribution homogène des algues dans toute la masse d'eau: dans ces conditions, l'évolution du taux de photosynthèse en profondeur est uniquement fonction de la pénétration de la lumière. Conformément au modèle proposé par Talling (1957) et Vollenweider (1965), l'activité photosynthétique par unité de surface de plan d'eau $(\Sigma A)$ peut être calculée selon la formule

$$
\Sigma A=\frac{A \max }{\alpha} \mathrm{f}(\mathrm{I})
$$

- La valeur du coefficient d'extinction vertical de la lumière $(\alpha)$ est essentiellement déterminée dans le Lot par la charge de matières organiques dissoutes (MOD) et de matières en suspension (MES). Les algues planctoniques ne représentent qu'une faible proportion des MES, si bien que les variations de biomasse ne se traduisent que par des changements peu importants de la profondeur de la zone euphotique ; cette dernière atteint 3,25 mètres en moyenne.

- L'activité photosynthétique du phytoplancton en lumière saturante (A max) varie avec le débit et la température, facteurs qui règlent l'évolution qualitative et quantitative de la biomasse d'algues.

La capacité photosynthétique du phytoplancton (Pmax) paraît dépendre essentiellement de la structure du peuplement : les Chlorophycées nanoplanctoniques se révèlent nettement plus efficaces que les Diatomées. Ce résultat rejoint les observations faites par d'autres auteurs (par exemple, Findenegg 1971). On sait également que Pmax dépend de la température, la valeur de $\mathrm{Q}^{10}$ avoisinant 2 (Talling 1966, Jewson 1976, Bindloss 1976). Dans le Lot, les températures élevées et les débits d'étiage coïncident avec le développement de Chlorophycées: les valeurs de Pmax s'accroissent pendant les trois mois d'été qui, au total, assurent près de $80 \%$ de la production annuelle.

- Les variations de $\mathrm{f}$ (I) suivent celles des deux variables $\mathrm{I}^{\prime}{ }_{0}$ et Ik. L'intensité de début de saturation par la lumière (Ik) est relativement élevée par rapport aux valeurs généralement trouvées dans la littérature (valeurs situées le plus souvent entre 1 et $2,5 \mathrm{cal} / \mathrm{cm}^{2} / \mathrm{h}$ ). La dif. férence tient en partie à la forte capacité photosynthétique du phytoplancton en été. Elle peut traduire également une adaptation des 
algues à la lumière, leur distribution homogène impliquant une circulation relativement rapide dans un fort gradient de lumière. L'existence de cette homogénéisation, condition nécessaire à l'utilisation du modèle précédent, amène les deux remarques suivantes :

1. - Les expériences réalisées dans cles flacons maintenus à un même niveau et non agités diffèrent des conditions naturelles; Mann et al. (1972) notent que, sur la Tamise, l'agitation des flacons pendant l'exposition augmente le taux de photosynthèse de près de $40 \%$. A l'inverse, Jewson et Wood (1975) montrent que les valeurs de $\Sigma$ A mesurées à l'aide de flacons en mouvement dans la zone euphotique diffèrent peu de celles calculées à partir d'expériences faites avec des flacons stationnaires.

2. - La diminution de $\mathrm{I}_{0}{ }_{0}$, l'augnentation de $\alpha$ ou celle de la profondeur moyenne de la rivière se traduisent par une diminution de la profondeur relative de la zone euphotique et, par suite, du temps passé par les algues à la lumière. Il en résulte un accroissement de la respiration et une diminution de la production nette. La comparaison de la croissance des algues et de l'assimilation photosynthétique de carbone dans une masse d'eau montre que $1 / 5^{\mathrm{e}}$ à peine du $\mathrm{C}$ assimilé sert à l'édification de biomasse nouvelle. En l'absence de zooplancton, les pertes sont attribuables à la respiration et à la décomposition bactérienne; les expériences montrent que la consommation d'oxygène à l'obscurité fournit peu d'indications sur l'intensité de ce processus, la minéralisation des matières organiques non algales (MOD et MOP) contribuant pour plus de $50 \%$ à la consommation moyenne d'oxygène.

Ces matières organiques sont en partie originaires du métabolisme du phytoplancton. Les expériences réalisées à Cajarc indiquent que le nanoplancton estival à base de Chlorophycées relargue des quantités importantes de matières organiques. A cette production extracellulaire est associée une activité hétérotrophe plus importante dont témoignent l'assimilation obscure du ${ }^{14} \mathrm{C}$ et les taux de consommation d' $\mathrm{O}_{2}$.

\section{TRAVAUX CITES}

ANDRÉ (E.). 1926. - Sur le plancton du Rhône. Rev. Hydrobiol., 3 : 159-266.

ARthur (C. R.) et Rigler (F. H.). 1967. - A possible source of error in the C14 method measuring primary productivity. Limnol. Oceanogr., 12 (1) : 121-124. BERNER (L. M.). 1951. - Limnology of the lower Missouri river. Ecology, 32 : 1-12. BINDLoss (M. E.). 1976. - The light-climate of loch Leven, a shallow Scottish lake, in relation to primary production by phytoplankton. Freshwat. Biol., 6: $501-518$.

Bordes (J.-M.), Lucchetta (J.-C.) et Rochard (M.). 1973. - Etude d'un écosystème d'eau courante : le Lot. Thèse $3^{c}$ cycle, Toulouse. $152 \mathrm{p}$. 
Brook (A.J.) et Rzoska (J.). 1954. - The influence of the Zebel Aulyia Dam on the development of Nile plankton. J. An. Ecol., 23 : 101-114.

Capblance (J.). 1972. - Phytoplancton et productivité primaire de quelques lacs d'altitude dans les Pyrénées. Annls Limnol., 8 (3) : 231-321.

Capblance (J.) et Décamps (H.). 1977. - Dynamics of the phytoplankton in the river Lot. Verh. Internat. Verein. Theor. Angew. Limnol., 20 (sous presse).

Capblance (J.) et Toureno (J. N.). 1978. - Hydrochimie de la rivière Lot. Annls Limnol., 14 (1-2) : 25-37.

ChоцNокy (B. J.). 1968. - Die ökologie der Diatomeen in Binnengewassern. J. Cramer Verlag. $700 \mathrm{p}$.

Cowel (B. C.). 1970. - The influence of plankton discharges from an upstream reservoir on standing crops in a Missouri river reservoir. Limnol. Oceanogr., $15: 426-441$.

C.T.G.R.E.F. 1974. - Etude de la productivité algale d'un site du Lot : le bief de Puy-l'Evêque. Rapport $n^{\circ} 2$ (non publié).

Cushing (C. E.). 1964. - Plankton and water chemistry in the Montreal river lake stream system, Saskatshewan. Ecology, $45:$ 306-313.

Czernin-Chudenitz (C. W.). 1958. - Limnologische Untersuchungen des Rheinstroms Bd III; Quantitative Phytoplanktonuntersuchungen. Forsch. Ber. Wirtschafts und Verkehrsministeriums Nordhein-Westfalen, $536: 1-224$.

Czernin-Chudenitz (C. W.). 1966. - Das phytoplankton Osterreichen Donau und seine Bedeutung fur die Selbstraining. Arch. Hydrobiol. Suppl., 30 : 194-217.

Dauta (A.). 1975. - Etude du phytoplancton du Lot. Annls Limnol., 11 (3) : 219. 238.

Denat (D.). 1977. - Etude de la production primaire dans le réservoir de Cajarc (Lot). Thèse $3^{\mathrm{e}}$ cycle, Toulouse. $94 \mathrm{p}$.

Des Cilleuls (J.). 1928. - Le phytoplancton de la Loire dans la région Saumuroise. Int. Rev. Ges. Hydrobiol. Hydrogr., 20 : 174-206.

DVinally (Z. T.). 1975. - Schätzung der Primärproduktion in Toten Donauarm Von Tolna und im Wasser des Sees Nagyszéktó von Kistelek. Symp. Biol. Hung., $15:$ 97-102.

EgboRge (A. B. M.). 1973. - A preliminary check list of the phytoplankton of the Oshum river, Nigeria. Freshwat. Biol., $3: 569-572$.

EGBORGE (A. B. M.). 1974. - The seasonal variation and distribution of phytoplankton in the river Oshum, Nigeria. Freshwat. Biol., $4: 177-191$.

Elster (H. J.). 1965. - Absolute and relative assimilation rates in relation to phytoplankton populations. Mem. Ist. Ital. Idrobiol. (suppl.), $18: 77-103$.

ERTL (M.) et JURIS (S.). 1967. - Measurements of primary production in the river Danube. Biol. Bratislava, 9: 654-658.

FEE (E. J.). 1973. - A numerical model for determining integral primary production and its application to lake Michigan. J. Fish. Res. Board. Can., 30 : 1467-1468.

FiNDENEGG (I.). 1965. - Relationship between standing crop and primary productivity. Mem. Ist. Ital. Idrobiol. (suppl.), $18:$ 271-289.

Findenegg (I.). 1971. - Die Produktionsleistungen einiger planktischer Algenarten in ihrem natürlichen Milieu. Arch. Hydrobiol., 69 : 273-293.

Germain (H.). 1936. - Diatomées d'eau douce. Thèse Sciences Bordeaux.

GolowiN (S.). 1971. - Comparison of pollution degree of fluvial waters evaluated by means of the $\mathrm{BOD}_{3}$ and saprobiological method. Polski Arch. Hydrobiol., 18 (4) : 381-391.

Golterman (H. L.). 1971. - The determination of mineralisation losses in correlation with the estimation of net primary production with the oxygen and chemical inhibitors. Freshwat. Biol., 1 : 249-256.

Greenberg (A. E.). 1964. - Plankton of the Sacramento river. Ecology, 45 : 40-49.

JAVORNICKY (P.). 1966. - Seasonal dynamics of the phytoplankton of Slapy reservoir, 1958-1960. Hydrobiol. Studies, 1 : 155-164. 
JEwson (D. H.). 1976. - The interaction of components controlling net phytoplankon photosynthesis in a well mixed lake (Lough Neagh, Northern Ireland). Freshwat. Biol., $6: 551-576$.

JEWSON (D. H.) et Wood (R. B.). 1975. - Some effects on integral photosynthesis of artificial circulation of phytoplankton through light gradients. Verh. Internat. Verein. Theor. Angew. Limnol., 19: 1037-1044.

Jones (D.) et WrLls (M. S.). 1956. - The attenuation of light in sea and estuarine waters in relation to the concentration of suspended solid matter. J. Mar. Biol. Ass. U. K., $35: 431-441$.

LACK (T. J.). 1971. - Quantitative studies on the phytoplankton of the river Thames and Kennet at Reading. Freshwat. Biol., $1: 213-224$.

LEAN (D.). 1972. - Radioisotope studies showing the role of phosphorus in the formation of large molecular weight organic compounds present in lake water. Proc. int. Meet. Humic substances. Nieuwersluis 1972. Pudoc. Wageningen : $159-170$.

LEAN (D.). 1973. - Movements of phosphorus between its biologically important forms in lake water. J. Fish. Res. Board. Can., $30: 525-536$.

LEFÈvRE (M.). 1943. - Contribution à la connaissance du phytoplancton du bassin de la Seine. Extr. Cahiers Commission bassin de la Seine $n^{\circ} 3$.

LiebmanN (H.). 1960 et 1962. - Handbuch der Frishwasser und abwasser Biologie, Band I (1146 p) et Band II (580 p). R. Oldemburg Verlag. Munchen.

LORENZEN (H.). 1967. - Determination of chlorophyll and pheopigments : spectrophotometric equations. Limnol. Oceanogr., 12: 343-346.

Manguin (E.). 1949. - Recherches sur l'origine du phytoplancton de la Seine. Cahiers de la commission du Bassin de la Seine, $n^{\circ} 11$.

ManN (K. H.), BritTon (R. H.), Kowalczewski (A.), Lack (T. J.), Mathews (C. P.) et Mc Donald (I.). 1972. - Productivity and energy flow at all trophic levels in the river Thames, England. Proceed. I.B.P.-U.N.E.S.C.O. Symposium on Productivity Problems of Freshwaters, Kazimierz-Dolny, Poland, May 6-12, $1970: 579-596$.

Marquis (D. A.) et Yelenosky (G.). 1962. - A chemical light meter for forest research station. Paper $n^{\circ} 165$. Northeastern Forest experiment. StationForest service. U.S. Department of Agriculture.

Nalewajko (C.). 1966. - Dry weight, ash and volume data for some freshwater planktonic algae. J. Fish. Res. Board. Can. 23 : 1285-1287.

Nalewajko (C.) et SchindleR (D. W.). 1976. - Primary production, extracellular release and heterotrophy in two lakes of the ELA, Northwestern Ontario. J. Fish. Res. Board. Can., 33 : 219-266.

Patten (B. C.). 1968. - Mathemetical models of plankton production. Int. Rev. Ges. Hydrobiol. Hydrogr., 53 : 357-408.

PIERRE (J. F.). 1972. - Etude hydrobiologique de la Meurthe. Contribution à l'écologie des populations algales. Ann. Hydrobiol., $3: 5-19$.

Popova (T. G.), Vershinin (N. V.), Blagovidova (L. A.), Setsko (R. I.) et ChaiKoUSKaIR (T. S.). 1972. - Biological Characteristics of first reservoirs on the $\mathrm{Ob}$ and Yenisey rivers. Verh. Internat. Verein. Limnol., $18: 872-876$.

Prouse (G. A.) et Talling (J. F.). 1958. - The seasonal growth and succession of plankton algae in the white Nile. Limnol. Oceanogr., $3: 222-238$.

RaI (H.). 1974. - Limnological observations on the different rivers and lakes in the Ivory Coast. Hydrobiologia, $44: 301-307$.

RoDHe (W.). 1964. - Effects of impoundment on water chemistry and plankton in lake Ransaren (Swedish Lapplands). Verh. Internat. Verein. Limnol., 15 : 437-443.

RoDHe (W.). 1965. - Standard correlations between pelagic photosynthesis and light. Mem. Ist. Ital. Idrobiol., (suppl.), $18: 365-381$. 
Schindler (D. W.). 1970. - Production of phytoplankton and zooplankton in Canadian Schield Lakes. Proceed. I.B.P.-U.N.E.S.C.O. Symposium on Productivity problems of Freshwaters. Kazimierz-Dolny, Poland, May 6-12 1970: 311-331.

Schindler (D. W.), Schmidt (R. V.) et REID (R. A.). 1972. - Acidification and bubbling as an alternative to filtration in determining phytoplankton production by the $\mathrm{C} 14$ method. J. Fish. Res. Board. Can., $29: 1627-1631$.

Srvko (T. N.), Kovalevskaja (R. Z.), Mikheeva (T. M.) et Ostapenia (A. P.). 1972. Biological transformation of organic matter in a river. Verh. Internat. Verein. Limnol., 18 : 756-760.

SMIth (E. L.). 1936. - Photosynthesis in relation to light and carbon dioxide. Proc. Nat. Acad. Sci. Wash., 22 : 504-511.

Soltero (R. A.), Gasperino (A. F.) et Graham (W. G.). 1975. - Cultural eutrophication of Long lake, Washington. Verh. Internat. Verein. Limnol., 19 : 17781789.

StrathmanN (R. R.). 1967. - Estimating the organic carbon content of phytoplankton from cell volume or plasma volume. Limnol. Oceanogr., 12: 411-418.

Szemes (G.). 1967. - Das phytoplankton der Donau. Limnologie der Donau. 5 : 158-179. E. Schweizerbart'sche Verlag. Stuttgart.

Talling (J. F.). 1957. - The phytoplankton population as a compound photosynthetic system. New. Phytol., $56: 133-149$.

Talling (J. F.). 1965. - The photosynthetic activity of phytoplankton in East African Lakes. Int. Rev. Ges. Hydrobiol. Hydrogr., 50: 1-32.

Talling (J. F.). 1966. - Photosynthetic behaviour in stratified and unstratified lake populations of a planktonic diatom. J. Ecol., 54 : 99-127.

TALLING (J. F.). 1971. - The underwater light climate as a controlling factor in the production ecology of freshwater phytoplankton. Mitt. Int. Verein. Theor. Angew. Limnol., 19 : 214-243.

Toureno (J. N.), Capblanco (J.) et Casanova (H.). 1978. - Bassin versant et hydrologie de la rivière Lot. Annls. Limnol., 14 (1-2) : 9-23.

TUfFery (G.). 1971. - Etude des communautés planctoniques de la Seine à l'aval de Paris. Bull. Français Pisc., 243 : 51-68.

Turoboyski (L.). 1966. - Travaux et études concernant les organismes indicateurs pour établir la valeur de l'eau. Verh. Internat. Verein. Limnol., 16: 841-844.

UTERMöHL (V. H.). 1958. - Zur Vervollkommung der quantitative phytoplankton methodik. Mitt. Intern. Verein. Theor. Angew. Limnol., 9: 1-38.

Villegas (I.) et De Giner (G.). 1973. - Phytoplankton as a biological indicator of water quality. Wat. Res., $7:$ 479-487.

VenKaTESWARLU (V.). 1969. - An ecological study of the algae of the river Mosi, Hyberabad (India), with special reference to the water pollution. Algal periodicity. Hydrobiologia, $34: 533-560$.

VollenWeider (R. A.). 1965. - Calculation models of photosynthesis depth curves and some implications regarding day rates estimates in primary production measurement. Mem. Ist. Ital. Idrobiol. (suppl.), 18: 427-457.

Vollenweider (R. A.). 1968. - Les bases scientifiques de l'eutrophisation des lacs et des eaux courantes sous l'aspect particulier du phosphore et de l'azote comme facteur d'eutrophisation. Rapport O.C.D.E. D.A.S./C.S.I. 68-27: 128 p.

Weber (C. I.) et MoORe (D. R.). 1967. - Phytoplankton, seston and dissolved organic carbon in the little Miami river at Cincinnati, Ohio. Limnol. Oceanos r., $21: 311-318$.

Whitford (L. A. I.) et Schumacker (G. J.). 1963. - Communities of algae in North Carolina streams and their seasonal relations. Hydrobiologia, 22 : 133-196.

Wright (J. C.). 1954. - The hydrobiology of Atwood lake, a flood control reservoir. Ecology, $35:$ 305-316.

Young (W. C.), Hannan (H. H.) et Tatum (J. W.). 1972. - The physico-chemical limnology of a stretch of the Guadalupe river, Texas, with five main stream impoundments. Hydrobiologia, $40: 297-319$. 